Article

\title{
Near UV-Vis and NMR Spectroscopic Methods for Rapid Screening of Antioxidant Molecules in Extra-Virgin Olive Oil
}

\author{
Giulia Vicario ${ }^{1,+}$, Alessandra Francini ${ }^{1,+}$, Mario Cifelli ${ }^{2}$, Valentina Domenici ${ }^{2, *(D)}$ \\ and Luca Sebastiani ${ }^{1, *}$ \\ 1 BioLabs, Institute of Life Science, Scuola Superiore Sant'Anna, Piazza Martiri della Libertà 33, \\ 56127 Pisa, Italy; g.vicario@santannapisa.it (G.V.); a.francini@santannapisa.it (A.F.) \\ 2 Chemistry and Industrial Chemistry Department, University of Pisa, Via Moruzzi 13, 56124 Pisa, Italy; \\ mario.cifelli@unipi.it \\ * Correspondence: valentina.domenici@unipi.it (V.D.); luca.sebastiani@santannapisa.it (L.S.); \\ Tel.: +39-050-221-9215 (V.D.); +39-050-883-070 (L.S.) \\ + Contributed equally.
}

Received: 16 November 2020; Accepted: 5 December 2020; Published: 8 December 2020

\begin{abstract}
Several spectroscopic techniques have been optimized to check extra-virgin olive oil quality and authenticity, as well as to detect eventual adulterations. These methods are usually complementary and can give information about different olive oil chemical components with bioactive and antioxidant properties. In the present work, a well-characterized set of extra-virgin olive oil (cultivar Frantoio) samples from a specific area of Tuscany (Italy) were investigated by combining near UV-Vis absorption spectroscopy, ${ }^{1} \mathrm{H}$ and ${ }^{13} \mathrm{C}$ nuclear magnetic resonance (NMR) to identify and quantify different chemical components, such as pigments, secoiridoids and squalene, related to the nutritional and quality properties of olive oils. Moreover, the pigmentation index of olives, organoleptic and sensory properties, total phenolic compound contents and the lipidic fractions of olive oils were investigated. The results obtained are, finally, compared and discussed in order to correlate several properties of both olives and olive oils with specific features of the cultivation area.
\end{abstract}

Keywords: extra-virgin olive oil; UV-Vis spectroscopy; ${ }^{1} \mathrm{H}$ NMR; ${ }^{13} \mathrm{C}$ NMR; pigments; secoiridoids; squalene; antioxidant properties; polyphenols

\section{Introduction}

Spectroscopic methods are now widely considered efficient tools for food in terms of oxidative stability and quality but, also, authentication, due to their high sensitivity, rapidness and possibility to perform analyses directly on the sample without previous treatments [1-3]. Additionally, compared with standard chromatographic methodologies, spectroscopic techniques are often less time-consuming and less expensive. For these reasons, in the last decade, many spectroscopic techniques were optimized and used in combination with multivariate techniques to food analysis in terms of chemical composition, trace contaminant determinations, food quality and authenticity and fraud identifications [4,5]. Recent advances in the application of spectroscopic analytical methods for food quality assessment were reported in a recent review by Hassoun et al. [6] focusing on the application of nondestructive spectroscopic monitoring and process optimization during the production of meat and fish. Similarly, new highly technological spectroscopic approaches were reviewed by Wang et al. [7] concerning the trace analysis of persistent organic pollutants in different complex matrices, such as food ones. A very interesting review about the state-of-the-art of nuclear magnetic resonance methods applied to the quality assessment of a large variety of food and agricultural products was just 
published [8]. All these considerations are also valid for the case of olive oil. Several recent researches based on different spectroscopies have been carried out in order to determine the olive oil quality and authenticity $[9,10]$. A recent review about rapid and innovative instrumental approaches for oil quality assessment was published by Valli et al. [11].

Among the different categories of olive oils, different quality levels have been attributed, which also correspond to different economic values. This is probably one of the main reasons why extra-virgin olive oil (EVOO) is the most adulterated food matrix. Different frauds and adulterations, such as those based on the addition of different kinds of seed oils, refined oil or lower grade of olive oils, have stimulated the development of rapid, nondestructive and relatively cheap methods, such as several spectroscopic methods reported in several works [12-14].

Among the different spectroscopic techniques, infrared (IR) $[15,16]$ and near infrared (near-IR) spectroscopies [17] have recently attracted much attention due to the very fast analyses and to the development of specific multivariate mathematical tools to rapidly detect typical olive oil adulterations [18]. These techniques are mostly centered on the identification and quantification of the fatty acid components and their derivatives $[16,19]$.

Other spectroscopies applied to the study of olive oil are related to the absorption of UV-Vi light (UV-Vis absorption spectroscopy) and to fluorescence emission in the UV-Vis region (fluorescence spectroscopy). This last technique was only recently used to investigate the quality and authenticity of olive oil, mainly focusing on the chlorophyll contents in fresh oils versus not-fresh oils [20] and to the emission spectral profiles due to the polyphenols present in olive oil [21]. UV-Vis absorption spectroscopy is a technique able to detect with relatively high sensitivity single pigment contents in the visible range (from $\sim 400 \mathrm{~nm}$ to $\sim 800 \mathrm{~nm}$ ) and other compounds present in olive oil, such as polyphenols, peroxides and other fatty acid derivatives due to the oxidation of olive oils, in the UV range (from $\sim 230 \mathrm{~nm}$ to $\sim 400 \mathrm{~nm}$ ). UV-Vis spectroscopy combined with multivariate analyses was used to quantify the adulteration of extra-virgin olive oils (EVOOs) with low-grade oils [22]. It was also combined with electronic nose to verify the EVOOs' geographical origin [23] and with other nonselective (near and mid-infrared spectroscopy) and selective techniques to characterize Protected Designation of Origin (PDO) olive oils [24]. This spectroscopic technique was also applied to thermal edible oil evaluation [25]. Recently, a novel method to identify and quantify the main pigment contents based on the deconvolution of near UV-Vis absorption spectra of olive oils was implemented [26-28] and applied to the study of EVOOs produced in different Mediterranean countries [10] and during different harvesting years [29]. The major advantages of near UV-Vis absorption spectroscopy for the quantification of pigments are the very fast analysis (few minutes), the very low expense of both instrument and analysis and the accuracy of pigment determinations comparable with conventional chromatographic techniques [10].

Finally, one of the most successful spectroscopies to characterize olive oils is nuclear magnetic resonance (NMR). In particular, ${ }^{1} \mathrm{H}$ and ${ }^{13} \mathrm{C}$ NMR techniques have been used to analyze both olive oil as it is (in the bulk) and olive oil extracts. These methods, in rapid development, are now considered valuable tools for olive oil analysis and characterization $[9,30]$ Other magnetic nuclei, such as ${ }^{31} \mathrm{P}$, were used to investigate adulterations in olive oils by NMR [31,32]. ${ }^{1} \mathrm{H}$ NMR spectra of olive oil analyzed without treatment or of its solvent extracts give information about all major and minor olive oil components [33-35]. A ${ }^{1} \mathrm{H}$ NMR spectral analysis was indeed applied as the target analysis for the detection and quantification of phenolic compounds, diacylglycerols and for fatty acid composition determinations $[9,34,36,37]$. It was also employed in metabolic profiling and fingerprinting in order to detect adulterations [38], to assess geographical origins [35,39,40] or to define the employed harvest method [41]. ${ }^{13} \mathrm{C}$ NMR spectroscopy was used mainly to provide valuable information about the acyl distribution and the acyl positional distribution of glycerol tri-esters in oils [40]. A combination of mono-dimensional and bi-dimensional NMR techniques were employed to follow the olive oil chain production [42], while multiple quantum NMR methods were optimized to explore the polyphenol contents in olive oil [43]. 
Considering the scarce literature about the combination of different analytical techniques to the in-depth characterization of the olive oil, quali-quantitative analyses using near-UV-Vis absorption and NMR spectroscopic methods can help to better understand the product composition and to bring innovation in the olive oil sector.

The aim of this study was to verify if spectroscopic techniques (often considered complementary to conventional methods) could discriminate the monovarietal EVOOs derived from a small geographical area (PDO "Terre di Siena") for the antioxidant molecule composition.

\section{Materials and Methods}

\subsection{Chemicals}

Petroleum ether, deuterated chloroform, methanol, hexane, acetonitrile, Folin-Ciocalteu's reagent, sodium carbonate, gallic acid, syringaldehyde (98\% purity), 1-methoxy-2-(2-methoxyethoxy) ethane and deuterated chloroform $\left(\mathrm{CDCl}_{3}\right)$ were purchased from Sigma-Aldrich (Milan, Italy). Water was purified by using a Milli-Q system (Merck-Millipore, Milan, Italy).

\subsection{Sampling Area}

The experimental orchard was in Southern Tuscany (Cetona, Italy). The farms participating in the ASIOL-BIOSi project (Applicazione di nuove Strategie e tecniche Innovative in OLivicoltura BIOlogica in provincia di Siena) provided olives and extra-virgin olive oil (EVOO) samples for sensory and chemical analyses. Three different locations were chosen: (1) $367-\mathrm{m}$ altitude, $42.891^{\circ} \mathrm{N} 11.941^{\circ} \mathrm{E}$, (2) $480-\mathrm{m}$ altitude $42.854^{\circ} \mathrm{N}, 11.821^{\circ} \mathrm{E}$ and (3) $340-\mathrm{m}$ altitude $42.918^{\circ} \mathrm{N}, 11.914^{\circ}$ E. The three selected farmers produced a monovarietal EVOO (cultivar Frantoio), characterized by a high yield and high content of phenols.

During the maturation stage of olives (between the beginning of June and the end of December), the area was characterized by a maximum temperature of $41^{\circ} \mathrm{C}$. Orchard management, including pruning, was conducted according to local commercial practice. Olive fruits (cultivar Frantoio) were sampled directly in the olive grown at the end of the ripening, just before the milling (13 December 2017) and were analyzed independently after $24 \mathrm{~h}$ from sampling. Fresh weight, pulp moisture, pulp fat content (Soxhlet extraction) and pigmentation index (Pi) [44] were determined. Olives were processed within $24 \mathrm{~h}$ from harvest in the same oil mill with a continuous cycle plant, which works without adding water, under controlled temperature with a maximum of $27^{\circ} \mathrm{C}$. EVOO samples were stored directly in dark glass bottles in a dark room at temperatures of $18-20^{\circ} \mathrm{C}$.

\subsection{EVOO Analyses}

Chemical and physical analyses for the classification of the produced oils established by the Commission Implementing Regulation (EU) No. 1348/2013 and by the PDO (Protected Denomination of Origin) specific regulation were done by the oil mill and external laboratories. All samples qualified for the PDO "Olio Terre di Siena" EVOO brand mark. Moreover, all samples respected the organic production regulation (European Council Regulation n. 834/2007).

Sensory analysis was carried out by a panel of 8 tasters according to the official standard (established by the International Olive Oil Council) on the 27 February 2018, and the results were organized in histograms.

Determination of total phenols content was done according Goldsmith et al. [45], with few modifications. Olive oils ( $5 \mathrm{~g}$ ) were added to $15 \mathrm{~mL}$ of $\mathrm{MeOH}: \mathrm{H}_{2} \mathrm{O}$ mixture $(80 / 20, v / v)$. After vortexing the samples for $2 \mathrm{~min}$, they were extracted in an ultrasonic bath for $15 \mathrm{~min}$ and centrifuged at $5600 \mathrm{rpm}$ for $30 \mathrm{~min}$ at $4{ }^{\circ} \mathrm{C}$ (Allegra 64R, Beckma Coulter, Milan, Italy). About $2 \mathrm{~mL}$ of the supernatant phase was filtered through a $0.25-\mu \mathrm{m}$ filter. An aliquot $(300 \mu \mathrm{L})$ of this filtered phase was added to $300 \mu \mathrm{L}$ of Folin-Ciocalteu's reagent and left to react for $2 \mathrm{~min} . \mathrm{Na}_{2} \mathrm{CO}_{3} 7.5 \%(2.4 \mathrm{~mL})$ was added to the previous mixture and then left to react for $1 \mathrm{~h}$ in the dark at room temperature. A blank $\mathrm{MeOH} / \mathrm{H}_{2} \mathrm{O}$ mixture 
$(80 / 20, v / v)$ was prepared in the same way. Absorbance was measured at $\lambda=760 \mathrm{~nm}$. A calibration curve was built using gallic acid as the standard (concentration between approximately 10 and $100 \mu \mathrm{g} / \mathrm{mL}$ ). Results were expressed as gallic acid equivalents (GAE) $\mathrm{g}^{-1}$ fresh weight (FW). Samples were analyzed in triplicate.

Near-UV-visible (UV-Vis) absorption spectra collection does not require any sample preparation, as they were analyzed directly in the bulk. EVOO samples were put in a transparent quartz cuvette with an optical path of $0.5 \mathrm{~cm}$. The spectra were recorded for each sample in triplicate between 220 and $800 \mathrm{~nm}$ by using a Jasco (Lecco, Italy) UV-Vis V-550 double-beam spectrophotometer; air was considered as the second beam background, and 1-nm resolution was used. The spectra acquired were normalized by subtracting the absorbance value at $\lambda=720 \mathrm{~nm}$, where the sample does not absorb, to all the point in the spectra, before the spectral deconvolution for pigment quantification [26]. The main pigments ( $\beta$-carotene, lutein and/or 9-cis-neoxantin, among carotenoids, and pheophytin $\mathrm{A}$ and $\mathrm{B}$ ) and their content evolution in time (at 48, 168, 230 and 286 days after pressing) were studied.

NMR experiments were performed using a Bruker (Milan, Italy) Avance DRX 400 spectrometer operating at a proton Larmor frequency of $401.36 \mathrm{MHz} .{ }^{1} \mathrm{H}$ NMR spectral analyses were performed both on EVOO samples in the bulk and sample extracts to analyze the dominant lipidic component and the secoiridoids fraction, respectively. For a direct EVOO sample analysis, $50 \mu \mathrm{L}$ of olive oil (without any internal standard) were added to $450 \mu \mathrm{L}$ of deuterated chloroform and put in a NMR tube. ${ }^{1} \mathrm{H}$ NMR spectra of EVOOs in the bulk were acquired with a $30^{\circ}$ pulse on the ${ }^{1} \mathrm{H}$ channel of 3.6 microseconds at $23 \mathrm{~W}$, with a relaxation delay of $1.5 \mathrm{~s}$ and an acquisition time (FID) of $2 \mathrm{~s}$ in 32-k points. Number of scans was 256. Acquired spectra were processed using the TOPSPIN 3.5 program: they were zero-filled to $64 \mathrm{~K}$, and the phase was corrected automatically, while the baseline was adjusted manually to optimize the signal integration. The peaks were selected manually, referring to the literature data $[46,47]$, and their area was determined through deconvolution in the region of interest because signals often overlap, so it was difficult to do an accurate integration without deconvolution. Samples were studied in triplicate. Spectra of the replicas were normalized (ACD Labs NMR Spectroscopy softwar (Frankfurt, Germany), and the relative fatty acid composition was determined according to Guillen et al. [48].

Olive oil extraction was necessary to separate the hydrophilic phenolic compounds from the fat fraction of EVOOs. Sample preparation was done according to Karkoula et al. [49], with some modifications. About $4 \mathrm{~g}$ of the sample were added to $20 \mathrm{~mL}$ of hexane and shaken three times for $30 \mathrm{~s}$, waiting $5 \mathrm{~min}$ between shakings. The result was a homogeneous phase, as the oil is soluble in hexane. The solution was mixed with $25 \mathrm{~mL}$ of acetonitrile, shaken three times for $30 \mathrm{~s}$, waiting for $10 \mathrm{~min}$ between shakings, and then left until the phase is separated for approximately $1 \mathrm{~h}$. Finally, the acetonitrile fraction was collected, $500 \mu \mathrm{L}$ of internal standard (syringaldehyde, IUPAC name 4-hydroxy-3,5-dimethoxybenzaldehyde) were added and this mixture was evaporated under nitrogen flow. The residue of this extraction procedure was dissolved in $500 \mu \mathrm{L}$ of deuterated chloroform $\left(\mathrm{CDCl}_{3}\right)$ and transferred in an NMR tube. Samples were extracted in triplicate for standard deviation evaluation on the quantitative analysis.

${ }^{1} \mathrm{H}$ NMR spectra of the EVOO extracts were recorded by using a $30^{\circ}$ pulse on the ${ }^{1} \mathrm{H}$ channel of $3.9 \mu$ s at $29.92 \mathrm{~W}\left(90^{\circ}\right.$ pulse is $\left.11.8 \mu \mathrm{s}\right)$, with an acquisition time for the FID of $2 \mathrm{~s}$ (32-K points zero-filled to $64 \mathrm{~K}$ before Fourier-transform) and a relaxation delay of $1.5 \mathrm{~s}$. Number of scans was 400 for a total of about $16 \mathrm{~min}$. The line broadening was set at $0.5 \mathrm{~Hz}$ for the FID Fourier-transform; the phase was adjusted automatically, while the baseline was corrected manually in the region of interest. Spectra were normalized. The peaks were selected manually, referring to the literature data $[48,49]$. The peaks area was determined through the deconvolution of the signals. Quantification of the identified compounds was done according the method proposed by Karkoula et al. [49,50].

${ }^{13} \mathrm{C}$ NMR analyses were performed by using about $500 \mathrm{mg}$ of olive oil dissolved in $500 \mu \mathrm{L}$ of chloroform. Fifty microliters of internal standard (1-methoxy-2-(2-methoxyethoxy) ethane, named also diglyme) for squalene quantification were added. The protocol and the internal standard were chosen 
according Nam et al. [51]. ${ }^{13} \mathrm{C}$ NMR spectra of EVOOs in the bulk were recorded by using a standard inverse-gated decoupling sequence: a $30^{\circ}$ pulse on the ${ }^{13} \mathrm{C}$ channel of $2.7 \mu$ s at $80.91 \mathrm{~W}\left(90^{\circ}\right.$ pulse was $8.22 \mu \mathrm{s})$ with an FID acquisition time of $0.6 \mathrm{~s}$ (64k points) and a relaxation delay d $1 \mathrm{of} 2.5 \mathrm{~s}$ in order to obtain a homogeneous magnetization recovery for all the signal in the spectrum. Proton decoupling (waltz $1690^{\circ}$ pulse train of $90 \mathrm{~ms}$ at $0.51 \mathrm{~W}$ ) was performed only during the ${ }^{13} \mathrm{C}$ FID acquisition in order to remove the proton-carbon interactions. No proton irradiation during d1 delay was used in order to avoid the NOE (Nuclear Overhauser Effect) on carbon nuclei. Number of scans was 12,000 for a total acquisition time of about $10 \mathrm{~h}$. Additionally, in this case, before the acquisition of all these spectra, lock, shim and tune were optimized. In this case, "auto-shim" was set. The spectra were zero-filled to $132 \mathrm{~K}$, and line broadening was set at $1 \mathrm{~Hz}$. Phase was adjusted automatically, while baseline was corrected manually. Peaks were selected manually, referring to the literature data [51,52]. Peak area was determined through the deconvolution of the signals. Squalene quantitative determination was done according Nam et al. [51].

\subsection{Statistical Analysis}

Data were expressed as means \pm standard deviation (SD) for the triplicate runs. Moreover, the coefficient of variation $(\mathrm{CV} \%)$ was determined in order to assess the precision and the repeatability of the method (quantitative NMR (qNMR)). The one-way ANOVA was then used to examine differences between the mean values. Bonferroni's multiple comparison test was used to establish the significance of the obtained differences $(p<0.05)$. Statistical analysis was performed in PRISM, GraphPad 5 (San Diego, CA, USA).

\section{Results}

\subsection{Olive Fruits Analysis}

Olive fruits used for the oil production presented fresh weight and pulp moisture (Figure 1) between 1.7 and $2.2 \mathrm{~g}$ and $42 \%$ and $49 \%$, respectively. Pulp fat content at the time of harvest was in the range of $20-27 \%$ of the total olive weight. The pigmentation index was lower in the olive group 2 compared to olive groups 1 and 3, but none of these differences was significant.

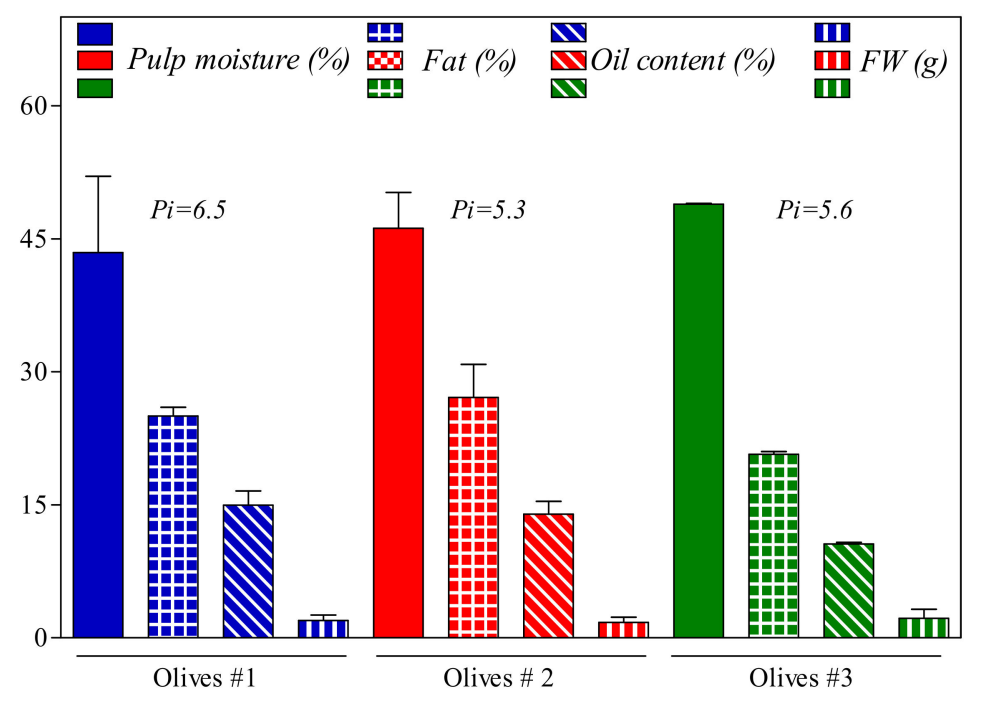

Figure 1. Change of some fruit physical and chemical characteristics of Frantoio olives harvested in three different location areas of Cetona (Tuscany, Italy). Pi: Pigmentation index; FW: fresh weight. 


\subsection{EVOO Analyses}

All the oils were obtained in the same oil mill, and the mandatory chemical analyses for "extra-virgin" category assignment were performed in the mill. Organoleptic analysis results were reported in Figure 2. No taste-olfactory defects were detected, confirming the "extra-virgin" category, but oils differ in terms of green fruitiness, bitter and pungent sensations and persistence of the aroma and taste. According to the descriptions, EVOO 2 is surely the most interesting: pungent and bitter notes are the most relevant perceptions, but also, a green fruitiness was detected. EVOO 1 and EVOO 3 show flatter profiles due to the reduced pungent/bitter gustative perceptions and the minor intensity of the fruitiness olfactory notes (green fruitiness was practically not relevant).

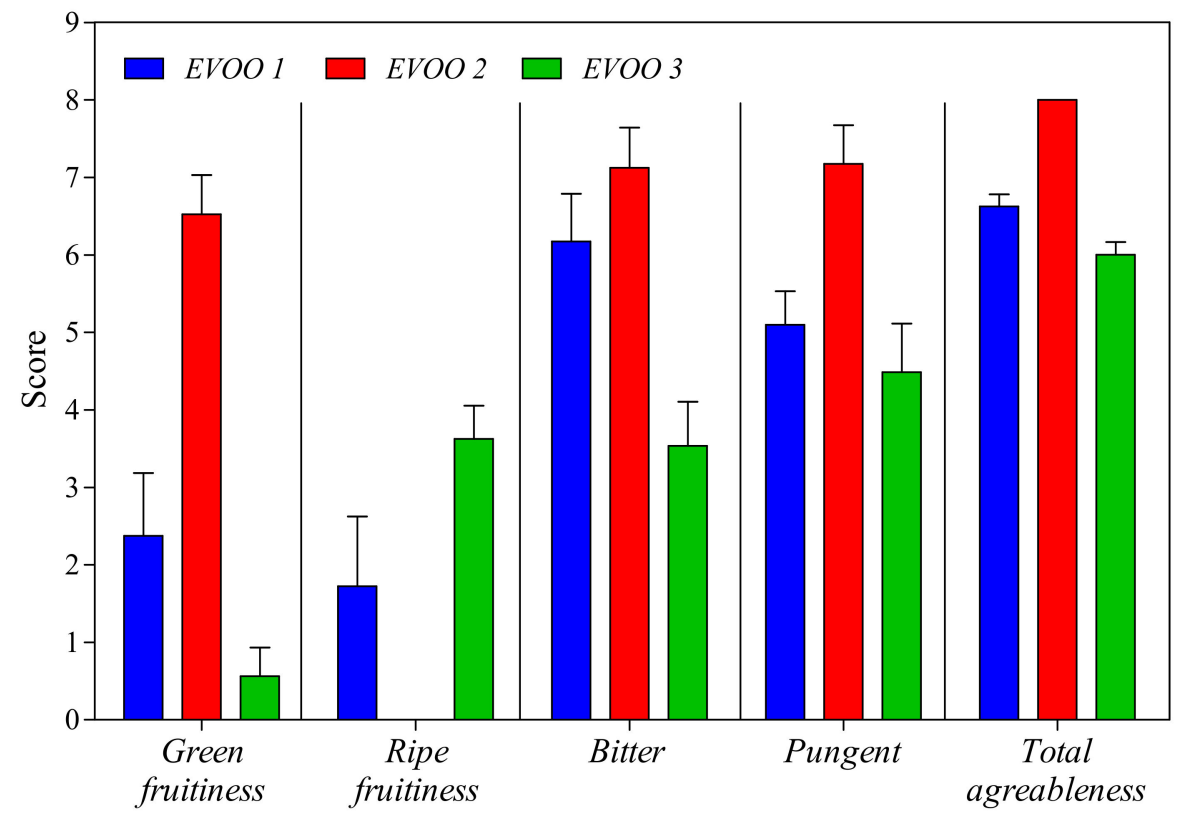

Figure 2. Bar plot of quantitative descriptive attributes of the three extra-virgin olive oils (EVOOs) studied.

As phenols are related to pungent and bitter perceptions and have important health properties, the total amount of phenols was determined by a Folin-Ciocalteu assay. The total phenols content results (Table 1) were significantly different in the EVOOs; EVOO 2 showed the highest content of total phenols compared to EVOO 1 and EVOO 3 and, in particular, $+35 \%$ and $+44 \%$, respectively.

Table 1. Total phenol contents in three different extra-virgin olive oils (EVOOs) $\left(\mathrm{mg} \mathrm{kg}^{-1}\right.$ fresh weight $(\mathrm{FW})$ ). Different letters represent differences following one-way ANOVA, Bonferroni post-test $p<0.05$.

Data represent means \pm standard deviation of three replicate.

\begin{tabular}{lc}
\hline & Total Phenols Content \\
\hline EVOO 1 & $129.64 \pm 3.22^{\mathrm{b}}$ \\
EVOO 2 & $200.88 \pm 1.90^{\mathrm{a}}$ \\
EVOO 3 & $111.03 \pm 2.38^{\mathrm{c}}$ \\
\hline
\end{tabular}

EVOO pigment contents are generally related to nutritional and stability properties. The acquisition of the near UV-Vis absorption spectra of EVOO samples is extremely fast (1 to $2 \mathrm{~min}$ ), and the quantitative analysis of the experimental spectra were performed by using a mathematical approach $[26,27]$. As expected, the near UV-Vis absorption spectral profiles (Figure 3) were inherent to extra-virgin olive oils and were similar for all the samples. However, considering the area below the curve and the absorbance values, it is evident also from a qualitative comparison that EVOO 2 contained 
more pigments with respect to EVOO 1 and EVOO 3. Among the various mathematical models tested, the best model for a quantitative description of EVOO pigments was the five-pigments (six-functions) model specific for olive oils after some months of storage (the R-square values obtained by using four different models are reported in the Supplementary Materials). This model allows the quantification of $\beta$-carotene, pheophytin-A, pheophytin-B, lutein and cis-neoxanthin, and it is very accurate (see Tables S1-S3 in the Supplementary Materials).

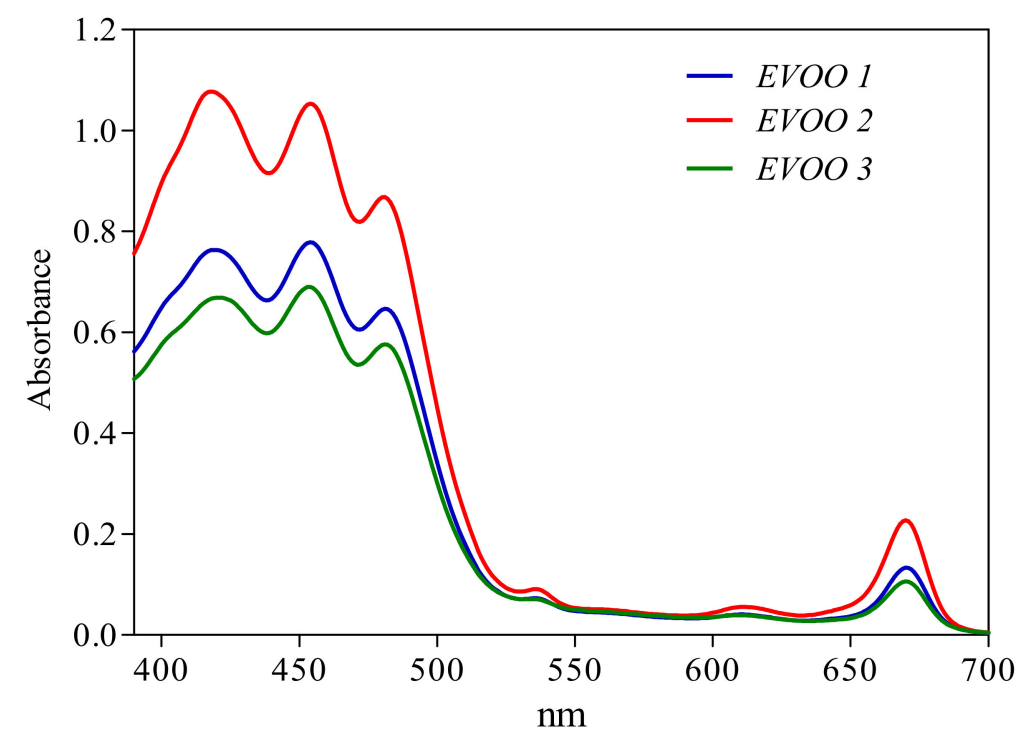

Figure 3. Near-UV-Vis absorption spectra of the three EVOO samples (EVOO 1: blue, EVOO 2: red and EVOO 3: green) in the range of wavelengths from 390 to $700 \mathrm{~nm}$.

The time evolution of pigment contents during the storage of the oil is reported in Figure 4 . After 48 days of storage (first sampling), the total pigment contents were significantly different in the three EVOOs of interest: EVOO 2 has higher amounts with respect to EVOO 1 and EVOO 3. With respect to the initial pheophytins fraction, EVOO 2 shows approximately double the amount of pheophytin A and pheophytin B with to respect EVOO 1 and EVOO 3, although the pheophytin A content was significantly different among the three samples. Considering carotenoids, no significant differences were detected in the $\beta$-carotene initial content, while cis-neoxanthin was more abundant in EVOO 2. Major differences can be observed in the lutein content. After 168 days of storage, total pigment contents were decreased in all the samples (-11\% for EVOO 1 and EVOO 2 and $-15 \%$ for EVOO 3). At the end of storage (286 days), losses in the pigments reached -17\% in EVOO 1 and $-16 \%$ in EVOO 2 and EVOO 3. The degradation of carotenoids and pheophytins occurred differently. Considering pheophytin A and pheophytin B (Figure 4C,D), in EVOO 1, we noted a reduction of $-12 \%$ and $-21 \%$, respectively, while, in EVOO 2 and EVOO 3, the reduction was considerably lower (-8\% and $-5 \%$, respectively, in EVOO2 and $-9 \%$ in EVOO 3). With respect to the carotenoids, the amount of lutein was gradually reduced during the storage, reaching $-17 \%,-21 \%$ and $-23 \%$ in EVOO 1, EVOO 2 and EVOO 3, respectively, at the end of the storage (Figure 4E). As concern $\beta$-carotene and cis-neoxanthin, the decrease did not have a linear trend differently from lutein, which represents the main carotenoid in olive oil. After 286 days, the $\beta$-carotene content was reduced by $-40 \%,-30 \%$ and $-15 \%$ in EVOO 1 , EVOO 2 and EVOO 3, respectively, but its amount in EVOO 3 behaved differently during the storage (Figure 4B). The cis-neoxanthin content, which is the minor carotenoid, was basically constant in EVOO 1 but consistently lower in EVOO 2 and EVOO 3 (-23\% and $-17 \%$, respectively) (Figure $4 \mathrm{~A})$. 

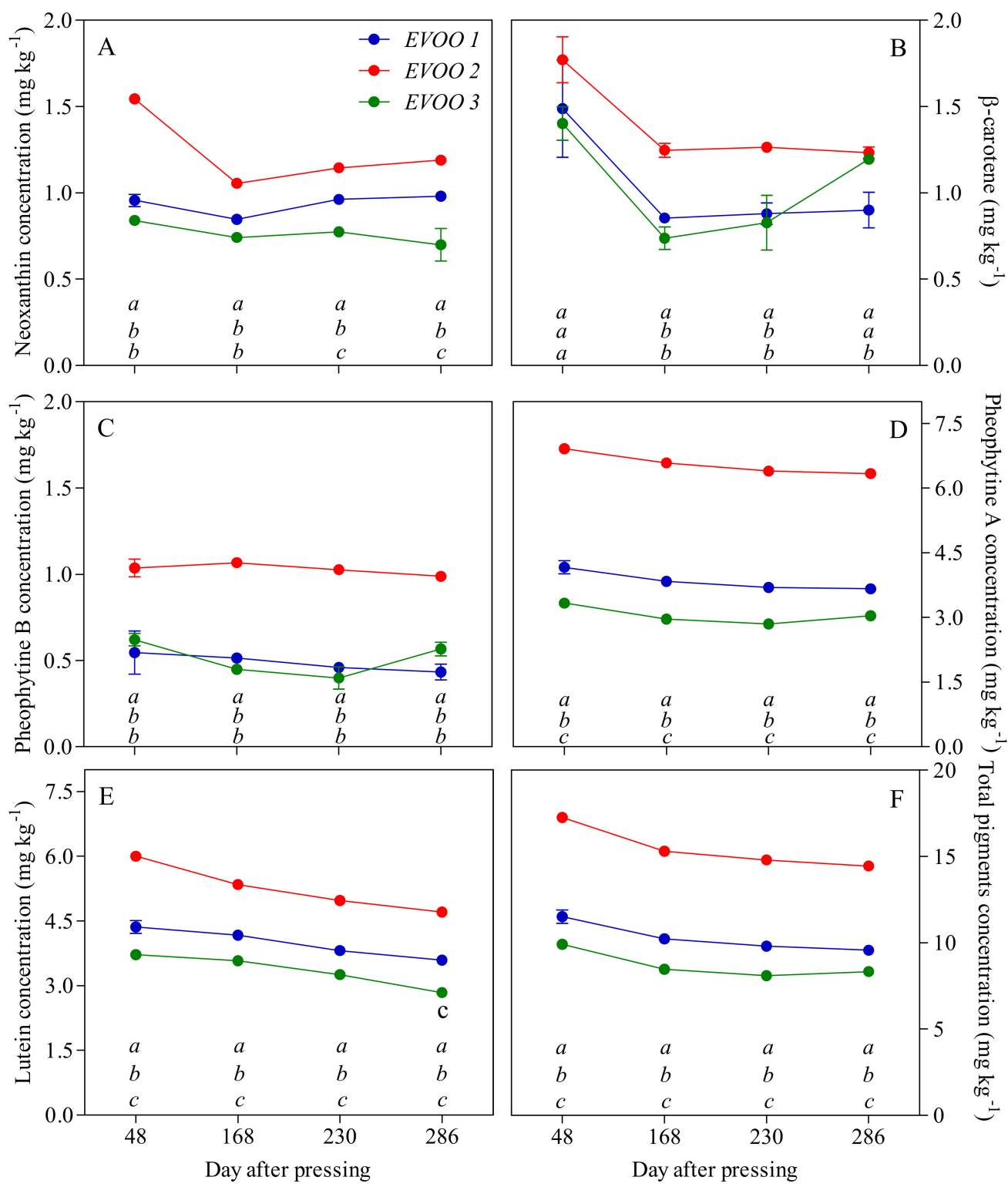

Figure 4. Pigment concentrations ( $\mathrm{mg} \mathrm{kg}^{-1}$ ) in EVOOs at different times of storage (days after pressing). (A) Neoxanthin; (B) $\beta$-carotene; (C) Pheophytin B; (D) Pheophytin A; (E) Lutein; (F) Total pigments concentrations. For each sampling data, different letters indicate significant differences among EVOOs. One-way ANOVA (Bonferroni post-test correction, $p<0.0001$ ).

In the middle of the storage period, the ${ }^{1} \mathrm{H} N M R$ spectra of EVOO samples in the bulk were acquired directly, dissolving the EVOO in deuterated chloroform. After normalization, the replicas of each sample showed the same spectral shape and the same proportions between signals, confirming the high repeatability of this ${ }^{1} \mathrm{H}$ NMR technique. The three EVOO spectra were overlapped in order to observe qualitative differences between the EVOOs of interest. Overall, qualitatively, it is possible to observe that the three EVOO samples are very similar in saponifiable fraction signals. Linolenic, linoleic, oleic and saturated acids were quantified according the formulas proposed by Guillen et al. [48] (see Table S4 in the Supplementary Materials). No significant differences were detected. Additionally, diacylglycerols signals appeared qualitatively similar in the three samples (see Figure S1 in the Supplementary Materials).

${ }^{1} \mathrm{H}$ NMR spectra of the EVOO extracts were acquired to determine the secoiridoid chemical composition. The ${ }^{1} \mathrm{H}$ NMR spectra of EVOO 1 and EVOO 3 appeared more similar than that of EVOO 2, 
especially concerning oleacein (identified by number 9 in Figure 5) and oleocanthal signals (identified by number 12 in Figure 5). EVOO 2 shows more intense oleuropein and ligstroside aglycones signals (identified in Figure 5 by the numbers 13 and 14, respectively) and a variety of not-yet-characterized signals between 9.3 and $9.4 \mathrm{ppm}$.

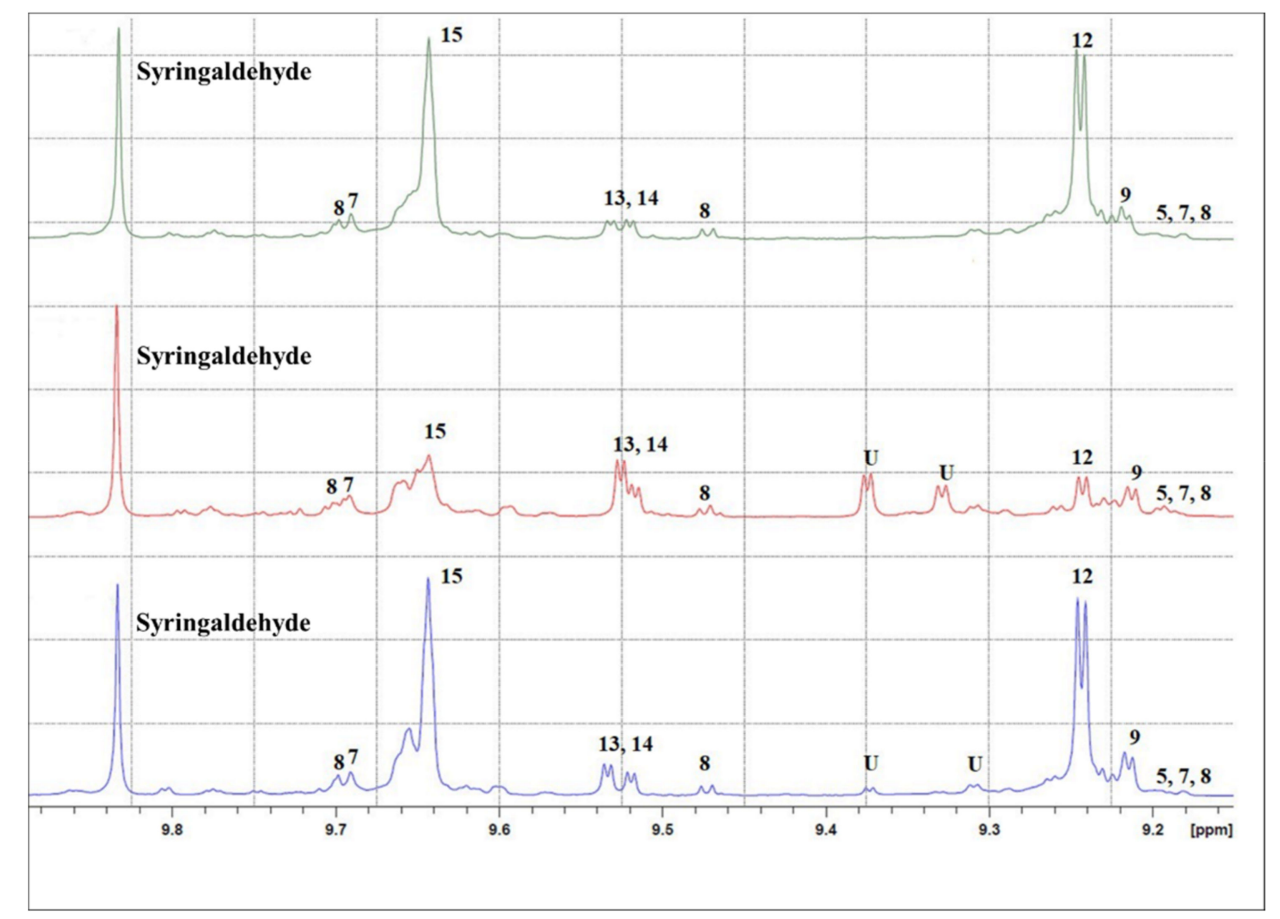

Figure 5. ${ }^{1} \mathrm{H}$ NMR spectra of EVOO 1 (blue), 2 (red) and 3 (green) in the 9.15-9.9 region. Syringaldehyde is the internal standard for quantification. Signals were identified according the literature data, and numeration refers to: (5) oleomissional, (7) 5S, 4R-oleuropeindial, (8) 5S, 4S-oleuropeindial, (9) oleacein (3,4-DHPEA-EDA), (12) oleocanthal (p-HPEA-EDA), (13) p-HPEA-EA (ligstroside aglycone), (14) 3,4-DHPEA-EA (oleuropein aglycone), (15) elenolic acid and $U$ = unknown signal. More details in Table S5.

Moreover, the squalene content was determined through the analysis of ${ }^{13} \mathrm{C}$ NMR spectra acquired in the bulk. Table 2 shows the results of the quantification of secoiridoids (oleacein, oleocanthal, oleuropein and ligstroside aglycones) and squalene.

Table 2. Oleacein, oleocanthal, oleuropein and ligstroside aglycones and squalene concentration $\left(\mathrm{mg} \mathrm{kg}^{-1}\right)$ in EVOO extracts, as obtained from ${ }^{1} \mathrm{H}$ NMR spectral analyses. Data are shown as mean $\pm \mathrm{SD}$. One-way ANOVA was performed (Bonferroni test correction $p<0.05$ ). For the same compound, different letters indicate differences among the EVOOs.

\begin{tabular}{lccc}
\hline & EVOO 1 & EVOO 2 & EVOO 3 \\
\hline Ligstroside aglycone & $27.9 \pm 1.43$ & $29.9 \pm 2.27$ & $25.4 \pm 1.23$ \\
Oleuropein aglycone & $39.6 \pm 2.05^{\mathrm{b}}$ & $67.8 \pm 1.80^{\mathrm{a}}$ & $26.2 \pm 2.52^{\mathrm{c}}$ \\
Total aglycones content & $67.5 \pm 2.25^{\mathrm{b}}$ & $97.7 \pm 2.56^{\mathrm{a}}$ & $51.5 \pm 1.56^{\mathrm{c}}$ \\
\hline Oleacein & $43.1 \pm 10.51$ & $31.0 \pm 4.37$ & $31.9 \pm 1.64$ \\
\hline Oleocanthal & $207.4 \pm 13.97^{\mathrm{a}}$ & $40.0 \pm 2.34^{\mathrm{b}}$ & $193.6 \pm 24.56^{\mathrm{a}}$ \\
\hline Squalene content & $3161 \pm 554$ & $2906 \pm 248$ & $2488 \pm 78$ \\
\hline
\end{tabular}

As expected, the squalene content was higher compared to other minor compounds and the quantification was quite accurate (maximum coefficient of variation $(\mathrm{CV})=17.8 \%$ ). No significant 
differences in squalene content were detected between the oils. Considering secoiridoids, the oleacein content was comparable between the oils, even if its quantification was the most variable considering the CV (24\%). Oleocanthal was more abundant in EVOO 1 and EVOO 3 compared with EVOO 2. The ligstroside aglycone content was comparable between oils, while oleuropein aglycone was higher in EVOO 2 followed by EVOO 1 and then EVOO 3. Oleocanthal and oleuropein/ligstroside aglycones determination was more accurate $(\mathrm{CV} \% \max =12.7 \%)$. The total aglycones content was also considered, because there is no agreement in the assignment of ligstroside and oleuropein aglycones signals. EVOO 2 showed the highest content in terms of total aglycones content.

\subsection{PCA Results}

A principal component analysis was performed to explore the data distribution patterns (total polyphenols, secoiridoids, squalene and pigments) and visualize the potential relationship among the studied variables and EVOOs. Figure 6 shows the bi-plot graph for the scores and loadings obtained. EVOO 1 and EVOO 3 obtained from olives with $\mathrm{Pi}=6.5$ and $\mathrm{Pi}=5.6$, respectively, are distributed on the top side of the graph, while the EVOO 2 obtained from olives with $\mathrm{Pi}=5.3$ is distributed on the bottom side of the graph. In order to detect important variables, a two-dimensional scatterplot of X-loadings for the first two components from the PCA is represented also in Figure 6. Similarly, Table 3 shows the loading factors of the PC1 and PC2 variables. The values with the greatest factor loading for PC1 are total polyphenols, oleocanthal, oleuropein-aglycon, neoxanthin, ligstroside-aglycon, $\beta$-carotene, pheophytin-B, pheophytin-A and lutein. Likewise, the variables showing a greater factor loading of the PC2 component were oleacein and squalene. The PCA analyses indicated that the compound oleacein is correlated with EVOO 1, while the attributes of total polyphenols, oleuropein-aglycon, neoxanthin, $\beta$-carotene, pheophytin $B$, pheophytin A and lutein are correlated with the EVOO 2. Finally, the data corresponding to the EVOO 3 sample did not cluster with any variable.

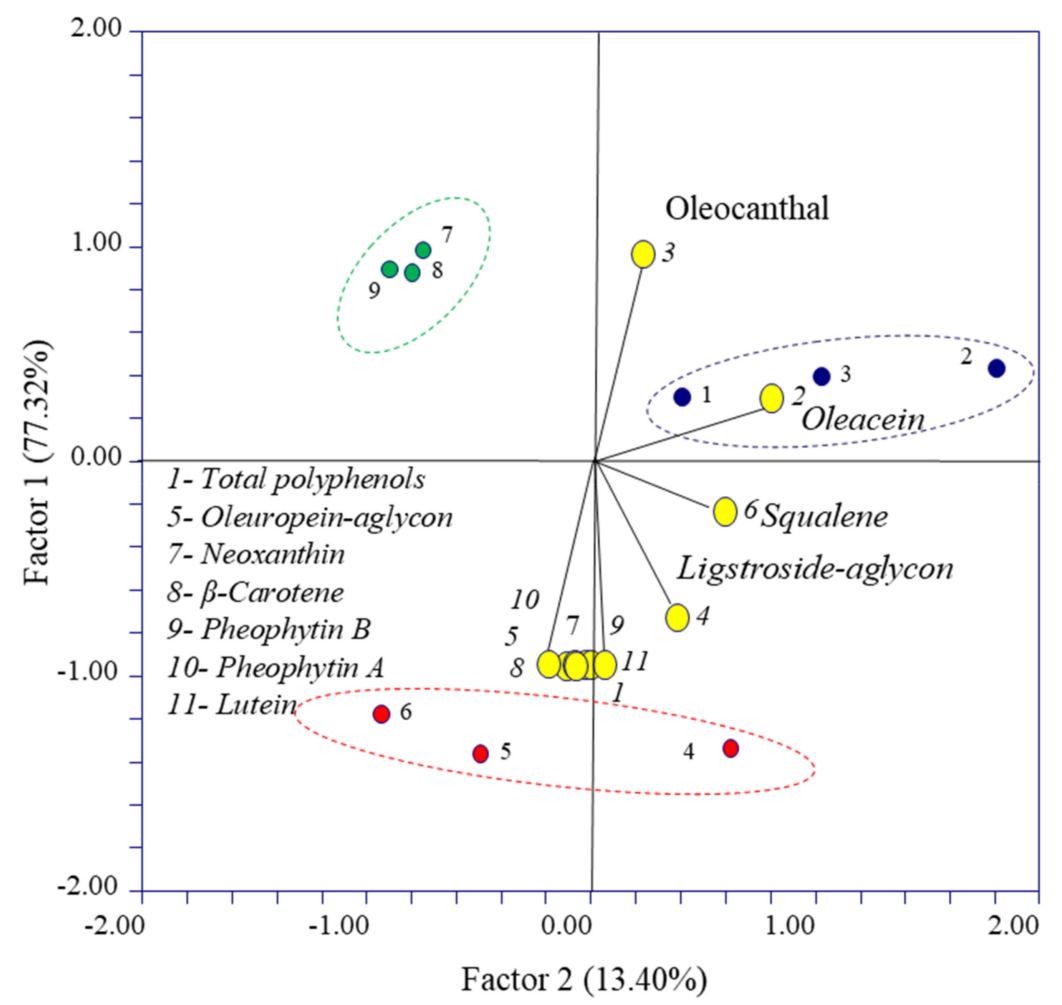

Figure 6. Bi-plot of the principal component analysis computed with the main results of the experiment. Data obtained from different EVOOs are reported with different colors: EVOO 1 blue, EVOO 2 red and EVOO 3 green. 
Table 3. X-loadings for the variables with respect to the principal components 1 and 2.

\begin{tabular}{llll}
\hline & Variables & PC1 & PC2 \\
\hline 1 & Total polyphenols & -0.998 & -0.051 \\
2 & Oleacein & 0.261 & 0.861 \\
3 & Oleocanthal & 0.937 & 0.289 \\
4 & Ligstroside-aglycon & -0.769 & 0.439 \\
5 & Oleuropein-aglycon & -0.990 & 0.037 \\
6 & Squalene & -0.270 & 0.650 \\
7 & Neoxanthin & -0.991 & 0.060 \\
8 & B-Carotene & -0.988 & -0.012 \\
9 & Pheophytin-B & -0.987 & -0.129 \\
10 & Pheophytin- $A$ & -0.997 & -0.007 \\
11 & Lutein & -0.991 & 0.084 \\
\hline
\end{tabular}

\section{Discussion}

EVOOs are food products that present a high variability of physical/chemical properties and their chemical and physical characterizations are usually performed by using different analytical methods. Several new and innovative techniques were recently optimized following the increased demand to replace traditional analytical methods with instrumental and, in particular, spectroscopic ones. The advantages to use spectroscopic methods are related to the speed of analysis, the possibility to analyze food matrices rich on antioxidant compounds without chemical or physical treatment and, in many cases, the high sensitivity combined with low cost. Chemical and food industries are looking forward to innovative instrumental techniques, such as spectroscopic methods for the qualitative and quantitative analyses of EVOOs. For instance, in the last years, the interest of a rapid and accurate quantification of pigments in the olive oil matrix has grown, and various spectrophotometric methods have been proposed [26,27]. Although analytical techniques aim to characterize the final product, the knowledge of the quality of olives at harvesting time, such as the area of cultivation and ripening stage, can help to make more accurate qualitative and quantitative analyses of EVOOs.

Considering the growing conditions, Mansour et al. [53] observed differences in the olive oil antioxidant chemical compositions of two local cultivars (Chemlali and Neb Jmel) grown in different areas: they detected higher olive oil total phenol contents at a higher altitude of cultivation. Similarly, Aguilera et al. [54] characterized EVOOs from the Frantoio cultivar grown in two different locations of Andalusia, showing that phenol contents in olive oils were higher at a higher altitude. In the present study, similar results were obtained from EVOO 2 that have been produced from olive groves located at $480 \mathrm{~m}$ above the sea level, while EVOO 1 and EVOO 3 derive from olive groves located at $367 \mathrm{~m}$ and $340 \mathrm{~m}$, respectively. This finding brings us to suppose that the different altitudes can influence the phenol EVOO composition.

Moreover, the slightly lower pigmentation index of olives of EVOO 2 detected at harvest time is in-line with the higher concentrations of total polyphenols detected in olive oil. As reported by Bengana et al. [55], there is an influence of olive ripeness on the chemical properties and phenolic composition of Chemlali extra-virgin olive oil. Chlorophylls and polyphenols tend to decrease in olive oil from advanced maturation stages of olives.

Some authors reported that olive ripeness has a strong impact on olive oil descriptors [56,57]. The small differences in pigmentation indices found among the three EVOOs in the present study are in agreement with the organoleptic properties. Data about green fruitiness highlights the higher score found in EVOO 2 with respect to the others. The literature reports that this attribute decreases with the ripening stage, and it tends to entirely disappear in the oil from fruits with high Pi [56]. Considering the pigments contribution to olive oil visual characteristics, many adulterations try to confuse consumers, mimicking the olive oil color by adding colorants [58]. Although is not our case, the near-UV-Vis method applied in this work has high potentialities for detecting adulterations of olive oil due to pigment additions. Moreover, chlorophylls and carotenoids are antioxidant 
compounds, and their quantification in olive oils stored in dark conditions is important for the quality and authenticity of EVOO samples. Furthermore, the near-UV-Vis spectroscopic method for EVOO analyses can also be useful to discriminate different olive oils. In this context, a recently published method [26,28] showed that the near-UV-Vis absorption spectrum of EVOO can be reproduced by the linear combination of four/five pure, mainly pigment spectral contributions. This method, applied to the present case, shows a general lower pigment content with respect to the data reported in the literature for EVOOs from the cultivar Frantoio [28], and this is probably due to the late harvest time of olives. Moreover, and more interesting, the total pigment contents were significantly different in the three EVOOs studied, EVOO 2 being the one with the highest, as also indicated by PCA analyses and in agreement with sensory properties of the EVOOs analyzed in this work.

Secoiridoids, like oleuropein and its derivates, belonging to the antioxidant class of polyphenols, have potential applications for several cancer treatments [59]. It is well-known that their amounts in EVOOs depend on many factors, such as the cultivar, ripening degree, pedoclimatic condition and irrigation. The ripening degree influences the oleuropein content; in fact, oleuropein increases during the first ripening stages (green olives) and, later, decreases significantly due to the polyphenol oxidase activity $[60,61]$. During ripening, the oleuropein decreases, because it is recycled to dimethyl oleuropein and elenolic acid glucoside [62]. Other interesting secoiridoid derivatives are aglycon forms of the secoiridoid glucosides produced during oil extraction [63]. A specific secoiridoid compound—namely, oleocanthal—firstly described by Montedoro et al. [64], is a promising antioxidant agent in the prevention of chronic diseases related to persistent conditions of inflammation and oxidative stress, attracting an increasing interest on its quantification $[65,66]$. Using ${ }^{1} \mathrm{H} \mathrm{NMR}$, the secoiridoid contents in the three EVOO samples were determined, showing that EVOO 2 has the highest amount of aglycon isomers of oleuropein (see Table 2), bioactive compounds associated with the bitter and pungent taste of olive oil $[67,68]$. This result agrees with the higher score of EVOO 2 of this parameter compared to EVOO 1 and EVOO 3, as reported in Figure 2.

Concerning oleocanthal, together with oleacin, its determination again discriminates EVOO 2 from the other two samples, EVOO 1 and EVOO 3, as reported in Table 3.

With the help of NMR spectroscopy, the olive oil matrix could also be directly investigated in order to assess the fatty acid profile, triacylglycerol composition, presence of diglycerides and trans-fatty acids, waxes and sterols profile $[33,69,70]$. In this context, we applied the method proposed by Nam et al. [51] for the direct quantification of squalene through ${ }^{13} \mathrm{C}$ NMR spectroscopy to our three EVOO samples. As shown in Table 2, no significant differences were revealed among the three EVOOs, but the amount of squalene detected in the present work are comparable to those found by Nam et al. [51] in the EVOO samples.

\section{Conclusions}

The presence of antioxidant molecules and their abundance in EVOOs strongly contributed to the organoleptic properties of the final product. The spectroscopic methods (UV-Vis and NMR) used in this work allowed to identify and quantify ten different olive oil chemical components with bioactive and antioxidant properties. In particular, five main pigments (neoxanthin, $\beta$-carotene, pheophytin-B, pheophytin-A and lutein) were determined and studied from the olive oil production to a long period of storage by using a fast and very cheap UV-Vis spectroscopic method. Four different secoiridoids (oleacein, oleocanthal, ligstroside-aglycon and oleuropein-aglycon) and squalene were quantified by using different NMR methods based on the ${ }^{1} \mathrm{H}$ and ${ }^{13} \mathrm{C}$ nuclei. Using compound types and abundance information, a discrimination analysis of monovarietal EVOOs derived from a small geographic area was achieved, proving the validity of spectroscopic methods for EVOO antioxidant identification.

Supplementary Materials: The following are available online at http://www.mdpi.com/2076-3921/9/12/1245/s1: Table S1, Table S2 and Table S3: Report the R-square obtained by fitting with four models the near-UV-Vis spectra of EVOO1, EVOO2 and EVOO3, respectively. Table S4: Reports the values of fatty acid components in the three EVOO samples. Table S5: Shows the assignment of the ${ }^{1} \mathrm{H}$ chemical shift of several compounds investigated in this 
work. Figure S1: Shows a selection of ${ }^{1} \mathrm{H}$ NMR spectra recorded in bulk (diacylglycerols signals) on the three EVOO samples.

Author Contributions: G.V., M.C. and V.D. contributed to the data collection and elaboration (biochemical, UV-Vis and NMR analyses and statistical elaboration); manuscript draft and its final approval. A.F. and L.S. contributed to the experiment planning, statistical analyses, manuscript draft and its final approval. All authors have read and agreed to the published version of the manuscript.

Funding: This research was funded by the ASIOLBIO-SI Progetto Integrato di Filiera (PIF) “Un filo d'oro: valorizzazione olio extra vergine di oliva di qualità certificata D.O.P. Terre di Siena e I.G.P. toscano in provincia di Siena" (CUP ARTEA 725734) project grant.

Acknowledgments: The authors wish to express their thanks to the financial support provided by the Agrobioscience PhD program at Scuola Superiore Sant'Anna of Pisa for granting G.V. scholarships and by the PiAnta project granted by Regione Toscana, POR FESR 2014-2020 for supporting the acquisition of part of the equipment used.

Conflicts of Interest: The authors declare no conflict of interest.

\section{References}

1. Afsah-Hejri, L.; Hajeb, P.; Ara, P.; Ehsani, R.J. A Comprehensive Review on Food Applications of Terahertz Spectroscopy and Imaging. Compr. Rev. Food Sci. Food Saf. 2019, 18, 1563-1621. [CrossRef]

2. Valand, R.; Tanna, S.; Lawson, G.; Bengtstrom, L. A review of Fourier Transform Infrared (FTIR) spectroscopy used in food adulteration and authenticity investigations. Food Addit. Contam. Part A 2020, 37, 19-38. [CrossRef] [PubMed]

3. McGrath, T.F.; Haughey, S.A.; Patterson, J.; Fauhl-Hassek, C.; Donarski, J.; Alewijn, M.; van Ruth, S.; Elliott, C.T. What are the scientific challenges in moving from targeted to non-targeted methods for food fraud testing and how can they be addressed-Spectroscopy case study. Trends Food Sci. Technol. 2018, 76, 38-55. [CrossRef]

4. Sorensen, K.M.; Khakimov, B.; Engelsen, S.B. The use of rapid spectroscopic screening methods to detect adulteration of food raw materials and ingredients. Curr. Opin. Food Sci. 2016, 10, 45-51. [CrossRef]

5. Lohumi, S.; Lee, S.; Lee, H.; Cho, B.K. A review of vibrational spectroscopic techniques for the detection of food authenticity and adulteration. Trends Food Sci. Technol. 2015, 46, 85-98. [CrossRef]

6. Hassoun, A.; Guðjónsdóttir, M.; Prieto, M.A.; Garcia-Oliveira, P.; Simal-Gandara, J.; Marini, F.; di Donato, F.; D'Archivio, A.A.; Biancolillo, A. Application of novel techniques for monitoring quality changes in meat and fish products during traditional processing processes: Reconciling novelty and tradition. Processes 2020, 8, 988. [CrossRef]

7. Wang, L.; Pang, S.; Zhou, G. Recent advances in spectroscopy technology for trace analysis of persistent organic pollutants. Appl. Sci. 2019, 9, 3439. [CrossRef]

8. Sobolev, A.P.; Thomas, F.; Donarski, J.; Ingallina, C.; Circi, S.; Marincola, F.C.; Capitani, D.; Mannina, L. Use of NMR applications to tackle future food fraud issues. Trends Food Sci. Technol. 2019, 91, 347-353. [CrossRef]

9. Dais, P.; Hatzakis, E. Quality assessment and authentication of virgin olive oil by NMR spectroscopy: A critical review. Anal. Chim. Acta 2013, 765, 1-27. [CrossRef]

10. Lazzerini, C.; Cifelli, M.; Domenici, V. Pigments in extra virgin olive oils produced in different Mediterranean countries in 2014: Near UV-vis spectroscopy versus HPLC-DAD. LWT Food Sci. Technol. 2017, 84, 586-594. [CrossRef]

11. Valli, E.; Bendini, A.; Berardinelli, A.; Ragni, L.; Ricco, B.; Grossi, M.; Gallina Toschi, T. Rapid and innovative instrumental approaches for quality and authenticity of olive oils. Eur. J. Lipid Sci. Technol. 2016, 118, 1601-1619. [CrossRef]

12. Meenu, M.; Cai, Q.; Xu, B. A critical review on analytical techniques to detect adulteration of extra virgin olive oil. Trends Food Sci. Technol. 2019, 91, 391-408. [CrossRef]

13. Ok, S. Detection of olive oil adulteration by low-field NMR relaxometry and UV-Vis spectroscopy upon mixing olive oil with various edible oils. Grasas Y Aceites 2017, 68, e173. [CrossRef]

14. Agiomyrgianaki, A.; Petrakis, P.V.; Dais, P. Detection of refined olive oil adulteration with refined hazelnut oil by employing NMR spectroscopy and multivariate statistical analysis. Talanta 2010, 80, 2165-2171. [CrossRef] [PubMed]

15. Mahesar, S.A.; Lucarini, M.; Durazzo, A.; Santini, A.; Lampe, A.I.; Kiefer, J. Application of Infrared Spectroscopy for Functional Compounds Evaluation in Olive Oil: A Current Snapshot. J. Spectrosc. 2019, 2019, 5319024. [CrossRef]

16. Nenadis, N.; Tsimidou, M.Z. Perspective of vibrational spectroscopy analytical methods in on-field/official control of olives and virgin olive oil. Eur. J. Lipid Sci. Tech. 2017, 119, 1600148. [CrossRef] 
17. Casale, M.; Simonetti, R. Review: Near infrared spectroscopy for analysing olive oils. J. Near Infrared Spectr. 2014, 22, 59-80. [CrossRef]

18. Gertz, C.; Matthaus, B.; Willenberg, I. Detection of Soft-Deodorized Olive Oil and Refined Vegetable Oils in Virgin Olive Oil Using Near Infrared Spectroscopy and Traditional Analytical Parameters. Eur. J. Lipid Sci. Technol. 2020, 122, 1900355. [CrossRef]

19. Sohng, W.; Park, Y.; Jang, D.; Cha, K.; Jung, Y.M.; Chung, H. Incorporation of two-dimensional correlation analysis into discriminant analysis as a potential tool for improving discrimination accuracy: Near-infrared spectroscopic discrimination of adulterated olive oils. Talanta 2020, 212, 120748. [CrossRef]

20. el Orche, A.; Bouatia, M.; Mbarki, M. Rapid analytical method to characterize the freshness of olive oils using fluorescence spectroscopy and chemometric algorithms. J. Anal. Met. Chem. 2020, 2020, 8860161. [CrossRef]

21. Ali, H.; Iqbal, M.A.; Atta, B.M.; Ullah, R.; Khan, M.B. Phenolic Profile and Thermal Stability of Monovarietal Extra Virgin Olive Oils Based on Synchronous Fluorescence Spectroscopy. J. Fluo. 2020, 30, 939-947. [CrossRef] [PubMed]

22. Torrecilla, J.S.; Rojo, E.; Domínguez, J.C.; Rodríguez, F.A. Novel Method To Quantify the Adulteration of Extra Virgin Olive Oil with Low-Grade Olive Oils by UV-Vis. J. Agric. Food Chem. 2010, 58, 1679-1684. [CrossRef] [PubMed]

23. Casale, M.; Armanino, C.; Casolino, C.; Forina, M. Combining information from headspace mass spectrometry and visible spectroscopy in the classification of the Ligurian olive oils. Anal. Chim. Acta 2007, 589, 89-95. [CrossRef] [PubMed]

24. Casale, M.; Oliveri, P.; Casolino, C.; Sinelli, N.; Zunin, P.; Armanino, C.; Forina, M.; Lanteri, S. Characterisation of PDO olive oil Chianti Classico by non-selective (UV-Visible, NIR and MIR spectroscopy) and selective (fatty acid composition) analytical techniques. Anal. Chim. Acta 2012, 712, 56-63. [CrossRef] [PubMed]

25. Gonçalves, R.P.; Março, P.H.; Valderrama, P. Thermal edible oil evaluation by UV-Vis spectroscopy and chemometrics. Food Chem. 2014, 163, 83-86. [CrossRef]

26. Domenici, V.; Ancora, D.; Cifelli, M.; Serani, A.; Veracini, C.A.; Zandomeneghi, M. Extraction of Pigment Information from Near-UV Vis absorption spectra of Extra Virgin Olive oils. J. Agric. Food Chem. 2014, 62, 9317-9325. [CrossRef]

27. Buti, F. Implementation of a Spectroscopic Method to Determine Pigments' Content in Olive Oil. Master's Thesis, University of Pisa, Pisa, Italy, 2016.

28. Borrello, E.; Domenici, V. Determination of pigments in virgin and extra-virgin olive oils: A comparison between two Near UV-Vis Spectroscopic Techniques. Foods 2019, 8, 18. [CrossRef]

29. Lazzerini, C.; Domenici, V. Pigments in extra-virgin olive oils produced in Tuscany (Italy) in different years. Foods 2017, 6, 25. [CrossRef]

30. Olmo-Cunillera, A.; Lopez-Yerena, A.; Lozano-Castellon, J.; Tresserra-Rimbau, A.; Vallverdu-Queralt, A.; Perez, M. NMR spectroscopy: A powerful tool for the analysis of polyphenols in extra virgin olive oil. J. Sci. Food Agric. 2020, 100, 1842-1851. [CrossRef]

31. Dais, P.; Spyros, A. $P^{31}$ NMR spectroscopy in the quality control and authentication of extra-virgin olive oil: A review of recent progress. Magn. Reson. Chem. 2007, 45, 367-377. [CrossRef]

32. Spyros, A.; Dais, P. Application of ${ }^{31}$ P NMR Spectroscopy in Food Analysis. 1. Quantitative determination of the mono- and diglyceride composition of olive oils. J. Agric. Food Chem 2000, 48, 802-805. [CrossRef] [PubMed]

33. Sacchi, R.; Mannina, L.; Fiordiponti, P.; Barone, P.; Paolillo, L.; Patumi, M.; Segre, A. Characterization of Italian extra virgin olive oils using $\mathrm{H}^{1}$ NMR spectroscopy. J. Agric. Food Chem. 1998, 46, 3947-3951. [CrossRef]

34. Barison, A.; Pereira da Silva, W.; Campos, F.R.; Simonelli, F.; Lenz, C.A.; Ferreira, A.G. A simple methodology for the determination of fatty acid composition in edible oils through ${ }^{1} \mathrm{H}$ NMR spectroscopy. Mang. Reson. Chem. 2010, 48, 642-650.

35. Mannina, L.; Patumi, M.; Proietti, N.; Bassi, D.; Segre, A.L. Geographical characterization of Italian extra virgin olive oils using high-field $\mathrm{H}^{1}$ NMR spectroscopy. J. Agri. Food Chem. 2001, 49, 2687-2696. [CrossRef] [PubMed]

36. Christophoridou, S.; Dais, P.; Tseng, L.H.; Spraul, M. Separation and identification of phenolic compounds in olive oil by coupling high-performance liquid chromatography with post-column solid-phase extraction to nuclear magnetic resonance spectroscopy (LC-SPE-NMR). J. Agric. Food. Chem. 2005, 53, 4667-4679. [CrossRef] [PubMed]

37. Perez-Trujillo, M.; Gomez-Caravaca, A.M.; Segura-Carretero, A.; Fernandez-Gutierrez, A.; Parella, T. Separation and Identification of Phenolic Compounds of Extra Virgin Olive Oil from Olea europaea L. by HPLC-DAD-SPE-NMR/MS. Identification of a new diastereoisomer of the aldehydic form of oleuropein aglycone. J. Agric. Food Chem. 2010, 58, 9129-9136. [CrossRef] [PubMed] 
38. Fauhl, C.; Reniero, F.; Guillou, C. ${ }^{1} \mathrm{H}$ NMR as a tool for the analysis of mixtures of virgin olive oil with oils of different botanical origin. Magn. Reson. Chem. 2000, 38, 436-443. [CrossRef]

39. Girelli, C.R.; del Coco, L.; Zelasco, S.; Salimonti, A.; Conforti, F.L.; Biagianti, A.; Barbini, D.; Fanizzi, F.P. Traceability of "Tuscan PGI" Extra Virgin Olive Oils by H NMR Metabolic Profiles Collection and Analysis. Metabolites 2018, 8, 60. [CrossRef]

40. Mannina, L.; Luchinat, C.; Patumi, M.; Emanuele, M.C.; Rossi, E.; Segre, A. Concentration dependence of $\mathrm{C}^{-13}$ NMR spectra of triglycerides: Implications for the NMR analysis of olive oils. Magn. Reson. Chem. 2000, 38, 886-890. [CrossRef]

41. D’Imperio, M.; Gobbino, M.; Picanza, A.; Costanzo, S.; Della Corte, A.; Mannina, L. Influence of harvest method and period on olive oil composition: An NMR and statistical study. J. Agric. Food Chem. 2010, 58, 11043-11051. [CrossRef]

42. del Coco, L.; de Pascali, S.A.; Iacovelli, V.; Cesari, G.; Schena, F.P.; Fanizzi, F.P. Following the olive oil production chain: 1D and 2D NMR study of olive paste, pomace, and oil. Eur. J. Lipid Sci. Technol. 2014, 116, 1513-1521. [CrossRef]

43. Reddy, G.N.M.; Mannina, L.; Sobolev, A.P.; Caldarelli, S. Polyphenols Fingerprinting in Olive Oils Through Maximum-Quantum NMR Spectroscopy. Food Anal. Methods 2018, 11, 1012-1020. [CrossRef]

44. Uceda, M.; Frias, L. Trend of the quality and quantitative composition of olive fruit oil during ripening. In Proceedings of the International Meeting on Olive Oil, Cordoba, Spain, 6-17 October 1975; pp. 25-46.

45. Goldsmith, C.D.; Stathopoulos, C.E.; Golding, J.B.; Roach, D.P. Fate of the phenolic compounds during olive oil production with the traditional press method. Int. Food Res. J. 2014, 21, 101-109.

46. Ruiz-Aracama, A.; Goicoechea, E.; Guillen, M.D. Direct study of minor extra-virgin olive oil components without any sample modification. ${ }^{1} \mathrm{H}$ NMR multisupression experiment: A powerful tool. Food Chem. 2017, 228, 301-314. [CrossRef]

47. Mannina, L.; Sobolev, A.P. High resolution NMR characterization of olive oils in terms of quality, authenticity and geographical origin. Magn. Reson. Chem. 2011, 49, S3-S11. [CrossRef]

48. Guillen, M.D.; Uriarte, P.S. Relationships between the evolution of the percentage in weight of polar compounds and that of the molar percentage of acyl groups of edible oils submitted to frying temperature. Food Chem. 2013, 138, 1351-1354. [CrossRef]

49. Karkoula, E.; Shantzari, A.; Melliou, E.; Magiatis, P. Direct Measurement of Oleocanthal and Oleacein Levels in Olive Oil by Quantitative ${ }^{1} \mathrm{H}$ NMR. Establishment of a New Index for the Characterization of Extra Virgin Olive Oils. J. Agric. Food Chem. 2012, 60, 11696-11703. [CrossRef]

50. Karkoula, E.; Skantzari, A.; Meliou, E.; Magiatis, P. Quantitative Measurement of Major Secoiridoid Derivatives in Olive Oil Using qNMR. Proof of the Artificial Formation of Aldehydic Oleuropein and Ligstroside Aglycon Isomers. J. Agric. Food Chem. 2014, 62, 600-607. [CrossRef]

51. Nam, A.M.; Bighelli, A.; Tomi, F.; Casanova, J.; Paoli, M. Quantification of Squalene in Olive Oil Using ${ }^{13} \mathrm{C}$ Nuclear Magnetic Resonance Spectroscopy. Magnetochemistry 2017, 3, 34. [CrossRef]

52. Merchak, N.; Silvestre, V.; Loquet, D.; Rizk, T.; Akoka, S.; Bejjani, J. A strategy for simultaneous determination of fatty acid composition, fatty acid position, and position-specific isotope contents in triacylglycerol matrices by C-13-NMR. Anal. Bioanal. Chem. 2017, 409, 307-315. [CrossRef]

53. Mansour, A.B.; Gargouri, B.; Flamini, G.; Bouaziz, M. Effect of agricultural sites on differentiation between Chemlali and Neb Jmel olive oils. Oleo Sci. 2015, 64, 381-392. [CrossRef] [PubMed]

54. Aguilera, M.P.; Beltrán, G.; Ortega, D.; Fernández, A.; Jiménez, A.; Uceda, M. Characterization of virgin olive oil of Italian olive cultivars: 'Frantoio' and 'Leccino', grown in Andalusia. Food Chem. 2005, 89, 387-391. [CrossRef]

55. Bengana, M.; Bakhouche, A.; Lozano-Sánchez, J.; Amir, Y.; Youyou, A.; Segura-Carretero, A.; Fernández-Gutiérrez, A. Influence of olive ripeness on chemical properties and phenolic composition of Chemlal extra-virgin olive oil. Food Res. Int. 2013, 54, 1868-1875. [CrossRef]

56. Jimenez-Herrera, B.; Rivas-Velasco, A.; Sanchez-Ortiz, A.; Lorenzo-Tovar, M.L.; Ubeda-Muñoz, M.; Callejon, R.; de Quirós, E.O.B. Influencia del proceso de maduración del fruto en la calidad sensorial de aceites de oliva virgen de las variedades Picual, Hojiblanca y Picudo. Grasas Aceites 2012, 63, 403-410. [CrossRef]

57. Rotondi, A.; Bendini, A.; Cerretani, L.; Mari, M.; Lercker, G.; Toschi, T.G. Effect of olive ripening degree on the oxidative stability and organoleptic properties of cv. Nostrana di Brisighella extra virgin olive oil. J. Agric. Food Chem. 2004, 52, 3649-3654. 
58. Fang, M.; Tsai, C.-F.; Wu, G.-Y.; Tseng, S.-H.; Cheng, H.-F.; Kuo, C.-H.; Hsu, C.-L.; Kao, Y.-M.; Shih, D.Y.; Chiang, Y.-M. Identification and quantification of $\mathrm{Cu}$-chlorophyll adulteration of edible oils. Food Addit. Contam. Part B Surveill 2015, 8, 157-162. [CrossRef] [PubMed]

59. Gorzynik-Debicka, M.; Przychodzen, P.; Cappello, F.; Kuban-Janowska, A.; Marino Gamazza, A.; Wozniak, M.; Gorska-Ponikowska, M. Potential Health Benefits of Olive Oil and Plant Polyphenols. Int. J. Mol. Sci. 2018, 19, 547. [CrossRef]

60. Cecchi, L.; Migliorini, M.; Cherubini, C.; Giusti, M.; Zanoni, B.; Innocenti, M.; Mulinacci, N. Phenolic profiles, oil amount and sugar content during olive ripening of three typical Tuscan cultivars to detect the best harvesting time for oil production. Food. Res. Int. 2013, 54, 1876-1884. [CrossRef]

61. Ortega-García, F.; Blanco, S.; Peinado, M.A.; Peragón, J. Polyphenol Oxidase and Its Relationship with Oleuropein Concentration in Fruits and Leaves of Olive (Olea europaea) cv. "Picual" Trees during Fruit Development. Tree Physiol. 2008, 28, 45-54.

62. Sánchez, R.; García-Vico, L.; Sanz, C.; Pérez, A.G. An aromatic aldehyde synthase controls the synthesis of hydroxytyrosol derivatives present in virgin olive oil. Antioxidants 2019, 8, 352. [CrossRef]

63. Barbieri, S.; Bendini, A.; Valli, E.; Gallina-Toschi, T. Do consumers recognize the positive sensorial attributes of extra virgin olive oils related with their composition? A case study on conventional and organic products. J. Food Compos. Anal. 2015, 44, 186-195. [CrossRef]

64. Montedoro, G.; Servilli, M.; Baldioli, M.; Selvaggini, R.; Miniati, E.; Macchioni, A. Simple and hydrolysable compounds in virgin olive oil. Spectroscopic characterizations of the secoiridoid derivatives. J. Agric. Food Chem. 1993, 41, 2228-2234. [CrossRef]

65. Pang, K.L.; Chin, K.Y. The biological activities of oleocanthal from a molecular perspective. Nutrients 2018, 10, 570. [CrossRef] [PubMed]

66. Segura-Carretero, A.; Curiel, J.A. Current disease-targets for oleocanthal as promising natural therapeutic agent. Int. J. Mol. Sci. 2018, 19, 2899. [CrossRef]

67. Mateos, R.; Cert, A.; Pérez-Camino, M.C.; García, J.M. Evaluation of virgin olive oil bitterness by quantification of secoiridoid derivatives. J. Am. Oil Chem. Soc. 2004, 81, 71-75. [CrossRef]

68. Servili, M.; Selvaggini, R.; Esposto, S.; Taticchi, A.; Montedoro, G.; Morozzi, G. Health and sensory properties of virgin olive oil hydrophilic phenols: Agronomic and technological aspects of production that affect their occurrence in the oil. J. Chromatogr. A 2004, 1054, 113-127. [CrossRef]

69. Vlahov, G. Application of NMR to the study of olive oils. Prog. Nucl. Magn. Reson. Spectrosc. 1999, 35, 341-357. [CrossRef]

70. Vlahov, G. ${ }^{13} \mathrm{C}$ nuclear magnetic resonance spectroscopy to determine olive oil grades. Anal. Chim. Acta 2006, 577, 281-287. [CrossRef]

Publisher's Note: MDPI stays neutral with regard to jurisdictional claims in published maps and institutional affiliations.

(C) 2020 by the authors. Licensee MDPI, Basel, Switzerland. This article is an open access article distributed under the terms and conditions of the Creative Commons Attribution (CC BY) license (http://creativecommons.org/licenses/by/4.0/). 